\title{
INTERACTION AND GENE ASSOCIATION AND DISPERSION IN DIALLEL CROSSES WHERE GENE FREQUENCIES ARE UNEQUAL
}

\author{
ANNE COUGHTREY and KENNETH MATHER \\ University of Southampton
}

Received 15.iii.69

\section{INTRODUGTION}

IN analysis of diallel crosses it has been shown (Jinks, 1954; Hayman, 1954) that the $W r / V r$ graph will be a line of unit slope if, inter alia, two assumptions hold. These assumptions are that genes at different loci are randomly distributed among the parents, and that there is no interaction between genes at different loci. Failure of either of these assumptions generally leads to departure from the rectilinear relation of $W r$ and $V r$.

Mather (1967) discussed the effects of complementary and duplicate interaction relationships on the rectilinearity of the $W r / V r$ graph, under conditions of equal frequencies of alleles at each locus. In this paper the discussion is generalised for all gene frequencies. In addition, the effects of association and dispersion, for all gene frequencies, are considered further in terms of a little-used parameter.

The notation used is that of Hayman and Mather (1955). The interaction parameters, however, are defined in the way that Van der Veen (1959) attributes to Smith and Robson and are as used by Mather (1967). All nine possible genotypes produced by combination of two pairs of alleles $A$ - $a$ and $B-b$ can be described in terms of eight quantities, where $d_{a}, d_{b}$ relate respectively to the differences between the homozygotes $A A$ - $a a$ and $B B-b b ; h_{a}, h_{b}$ to the effects of dominance at the two loci; $i_{a b}$ to the interaction between the two loci when both are homozygous; $j_{a / b}$ and $j_{b / a}$ to the interaction between $d_{a}$ and $h_{b}$, and $d_{b}$ and $h_{a}$ respectively; and $l_{a b}$ to the interaction between the two loci when both are heterozygous. The nine phenotypes are shown in terms of these quantities in table 1.

\section{INTERAGTION}

If the frequencies of $A, a$ at one locus are represented by $u_{a}, v_{a}\left(=1-u_{a}\right)$ respectively, and of $B, b$ at another locus by $u_{b}, v_{b}\left(=1-u_{b}\right)$, the results of a diallel cross of the four possible homozygous lines can be represented as in table 2, assuming that the genes under consideration are independently distributed among the parents. Using this table the variances and covariances can be found for each array, together with the mean variance and covariance of arrays, the variance and covariance of array means, and the variance of the parental lines for all gene frequencies. These are set out in table 3 where, for convenience $i_{a b}$ is written as $i, j_{a b}$ as $j_{a}, j_{b a}$ as $j_{b}$ and $l_{a b}$ as $l$.

For the classical situations of full complementary and duplicate interaction to exist, the eight parameters must all be of equal size and take characteristic signs. These are shown in table 4 which is taken from Mather's (1967) table 2. Thus, in the complementary relationship $d$ and $j$ are both 
positive, and $h, i$ and $l$ are all of the same sign, either positive or negative; in the duplicate relationship $j$ is negative, and $i$ and $l$ are of opposite sign to $h$.

If for simplicity the interaction parameters are given the same sign (as in the complementary situation $(a)$ and duplicate situation $(a)$ of table 4 )

\section{TABLE 1}

The 9 possible phenotypes produced by combination of 2 pairs of alleles $\mathrm{A}-\mathrm{a}$ and $\mathrm{B}-\mathrm{b}$

$$
\begin{array}{lrcc} 
& A A & A a & a a \\
B B & d_{a}+d_{b}+i_{a b} & h_{a}+d_{b}+j_{b a} & -d_{a}+d_{b}-i_{a b} \\
B b & d_{a}+h_{b}+j_{b} & h_{a}+h_{b}+l_{a b} & -d_{a}+h_{b}-j_{a b} \\
b b & d_{a}-d_{b}-i_{a b} & h_{a}-d_{b}-j_{b a} & -d_{a}-d_{b}+i_{a b}
\end{array}
$$

and in size equal to a quantity, $m$, the expressions for array variances and covariances in table 3 reduce to those shown in table 5. For full complementary and duplicate interaction all parameters are of equal size i.e. $d=h= \pm m$ and the expressions reduce still further (as shown in the lower part of table 5). It should be noted that situations $(b)$ of table 4 give equivalent results but with the arrays reversed.

TABLE 2

Phenotypes and array means of parents and progeny of a diallel cross of four homozygous lines

\begin{tabular}{|c|c|c|c|c|}
\hline $\begin{array}{l}\text { Parental } \\
\text { Line }\end{array}$ & $A A B B$ & $A A b b$ & $a a B B$ & $a a b b$ \\
\hline $\begin{array}{l}\text { Phenotype } \\
\text { Frequency }\end{array}$ & $\begin{array}{l}d_{a}+d_{b}+i_{a b} \\
u_{a} u_{b}\end{array}$ & $\begin{array}{l}d_{a}-d_{b}-i_{a b} \\
u_{a} v_{b}\end{array}$ & $\begin{array}{l}-d_{a}+d_{b}-i_{a b} \\
v_{a} u_{b}\end{array}$ & $\begin{array}{l}-d_{a}-d_{a}+i_{a b} \\
v_{a} v_{b}\end{array}$ \\
\hline $\begin{array}{l}A A B B \\
\quad\left(u_{a} u_{b}\right)\end{array}$ & $d_{a}+d_{b}+i_{a b}$ & $d_{a}+h_{b}+j_{a b}$ & $h_{a}+d_{b}+j_{b a}$ & $h_{a}+h_{b}+l_{a b}$ \\
\hline $\begin{array}{l}A A b b \\
\quad\left(u_{a} v_{b}\right)\end{array}$ & $d_{a}+h_{b}+j_{a b}$ & $d_{a}-d_{b}-i_{a b}$ & $h_{a}+h_{b}+l_{a b}$ & $h_{a}-d_{b}-j_{b a}$ \\
\hline $\begin{array}{l}a a B B \\
\left(v_{a} u_{b}\right)\end{array}$ & $h_{a}+d_{b}+j_{b a}$ & $h_{a}+h_{b}+l_{a b}$ & $-d_{a}+d_{b}-i_{a b}$ & $-d_{a}+h_{b}-j_{a b}$ \\
\hline $\begin{array}{l}a a b b \\
\quad\left(v_{a} v_{b}\right)\end{array}$ & $h_{a}+h_{b}+l_{a b}$ & $h_{a}-d_{b}-j_{b a}$ & $-d_{a}+h_{b}-j_{a b}$ & $-d_{a}-d_{b}+i_{a b}$ \\
\hline Array means & $\begin{array}{r}u_{a} d_{a}+u_{b} d_{b} \\
+v_{a} h_{a}+v_{b} h_{b}\end{array}$ & $\begin{array}{r}u_{a} d_{a}-v_{b} d_{b} \\
+v_{a} h_{a}+u_{b} h_{b}\end{array}$ & $\begin{array}{l}-v_{a} d_{a}+u_{b} d_{b} \\
-u_{a} h_{a}+v_{b} h_{b}\end{array}$ & $\begin{array}{l}-v_{a} d_{a}-v_{b} d_{b} \\
+u_{a} h_{a}+u_{b} h_{b}\end{array}$ \\
\hline & $\begin{array}{l}+u_{a} u_{b} i_{a b}+u_{a} v_{b} j_{a b} \\
+u_{b} v_{a} j_{b a}+v_{a} v_{b} l_{a b}\end{array}$ & $\begin{array}{l}-u_{a} v_{b} i_{a b}+u_{a} u_{b} j_{a b} \\
+v_{a} v_{b} j_{b a}+v_{a} u_{b} l_{a b}\end{array}$ & $\begin{array}{l}-v_{a} u_{b} i_{a b}-v_{a} v_{b} j_{a b} \\
+u_{a} u_{b} j_{b a}+u_{a} v_{b} l_{a b}\end{array}$ & $\begin{array}{l}+v_{a} v_{b} i_{a b}-v_{a} u_{b} j_{a b} \\
-u_{a} v_{b} j_{b a}+u_{a} u_{b} l_{a b}\end{array}$ \\
\hline
\end{tabular}

$\begin{aligned} \text { Mean of all progeny }= & \left(u_{a}-v_{a}\right) d_{a}+\left(u_{b}-v_{b}\right) d_{b}+2 u_{a} v_{a} h_{a}+2 u_{b} v_{b} h_{b} \\ & +\left(u_{a}-v_{a}\right)\left(u_{b}-v_{b}\right) i_{a b}+2 u_{b} v_{b}\left(u_{a}-v_{a}\right) j_{a b}+2 u_{a} v_{a}\left(u_{b}-v_{b}\right) j_{b a} \\ & +4 u_{a} v_{a} u_{b} v_{b} l_{a b} \\ \text { Mean of parents }= & \left(u_{a}-v_{a}\right) d_{a}+\left(u_{b}-v_{b}\right) d_{b}+\left(u_{a}-v_{a}\right)\left(u_{b}-v_{b}\right) i_{a b} .\end{aligned}$

It is apparent, from table 5 , that in the full complementary situation, assuming that $A, B$ are dominant to $a, b$ (i.e. that $d=h=m$, situation (a) of table 4) the point for array (1) lies at the origin, while that for array (4) lies on a line of unit slope passing through the origin. The points for arrays (2) and (3)) lie below this line (since $W r: V r=v_{b}: 1$ for array (2), and $v_{a}: 1$ for array (3) giving a graph which is characteristically concave upwards, as shown by Mather (1967) to apply in the special case where $u_{a}=u_{b}=v_{a}=v_{b}=\frac{1}{2}$. If the distance between the points for arrays (1) 


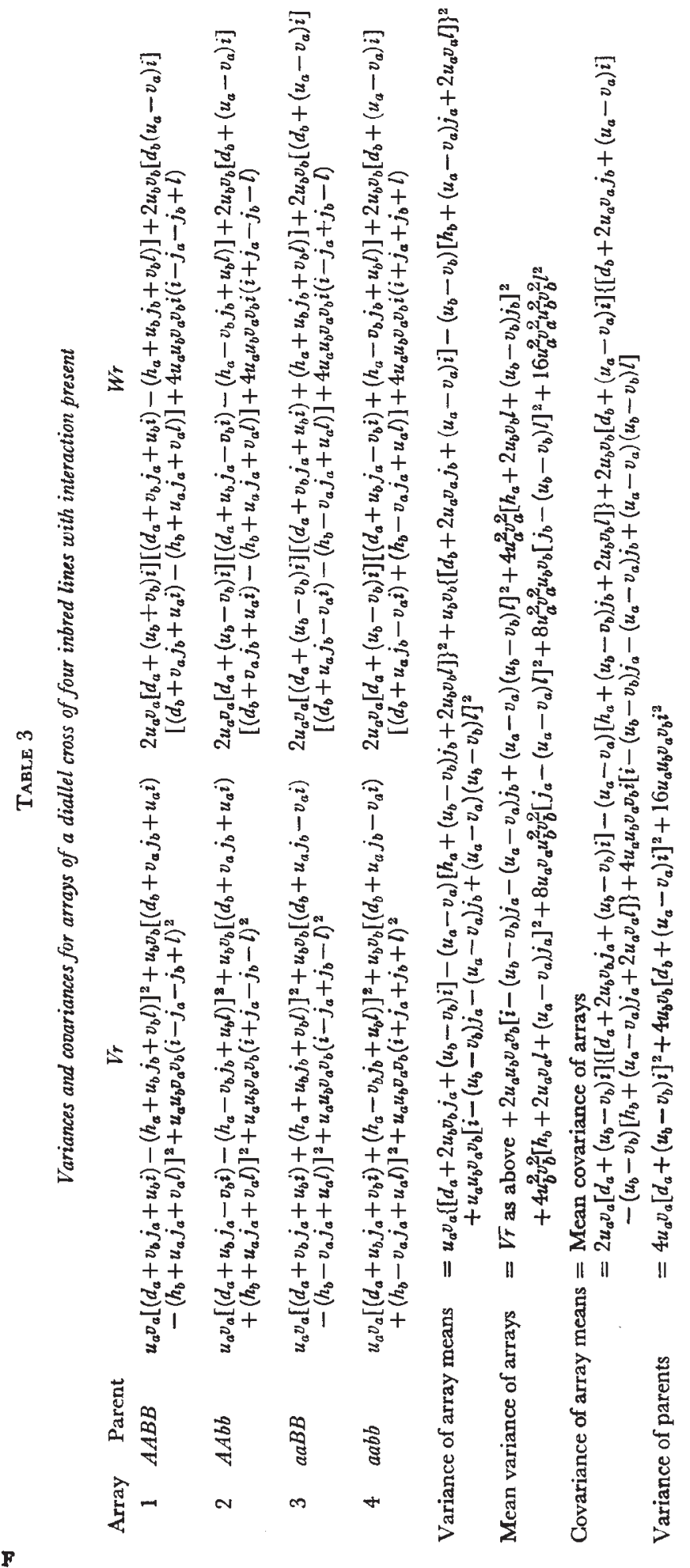


and (4) is then standardised for all gene frequencies (by varying the size of $m$ ) a series of curves is obtained, for different gene frequencies, as shown in fig. 1. As $u_{a}, u_{b}$ tend to 1 (i.e. there is an excess among the parents of the dominant alleles) so the curve approximates more closely to the line of unit slope.

\section{TABLE 4}

Signs of the 8 quantities in complementary and duplicate interaction relationships. Situations (a) and (b) for each are equivalent

$\begin{array}{lccccccccc} & & d_{a} & d_{b} & h_{a} & h_{b} & i & j_{a} & j_{b} & l \\ \text { Complementary }(a) & + & + & + & + & + & + & + & + \\ & (b) & + & + & - & - & - & + & + & - \\ \text { Duplicate } & (a) & + & + & + & + & - & - & - & - \\ & (b) & + & + & - & - & + & - & - & +\end{array}$

For full duplicate interaction a very different situation pertains. Where $A, B$ are the dominant alleles, the points for arrays (1), (2) and (3) all lie at the origin, while that for array (4) again lies on a line of unit slope passing through the origin. This is true for all gene frequencies.

\section{TABLE 5}

Variances and covariances of arrays for $\mathrm{i}=\mathrm{j}=1=\mathrm{m}$

\begin{tabular}{ccc} 
Array & \multicolumn{1}{c}{$V r$} & $W r$ \\
1 & $u_{a} v_{a}\left(d_{a}-h_{a}\right)^{2}$ & \\
& $+u_{b} v_{b}\left(d_{b}-h_{b}\right)^{2}$ & $2 u_{a} v_{a}\left(d_{a}+\left[u_{b}-v_{b}\right] m\right)\left(d_{a}-h_{a}\right)$ \\
2 & $u_{a} v_{a}\left(d_{a}-h_{a}\right)^{2}$ & $+2 u_{b} v_{b}\left(d_{b}+\left[u_{a}-v_{a}\right] m\right)\left(d_{b}-h_{b}\right)$ \\
& $+u_{b} v_{b}\left(d_{b}+h_{b}+2 m\right)^{2}$ & $2 u_{a} v_{a}\left(d_{a}+\left[u_{b}-v_{b}\right] m\right)\left(d_{a}-h_{a}\right)$ \\
& & $+2 u_{b} v_{b}\left(d_{b}+\left[u_{a}-v_{a}\right] m\right)\left(d_{b}+h_{b}+2 m\right)$ \\
3 & $u_{a} v_{a}\left(d_{a}+h_{a}+2 m\right)^{2}$ & $2 u_{a} v_{a}\left(d_{a}+\left[u_{b}-v_{b}\right] m\left(d_{a}+h_{a}+2 m\right)\right.$ \\
& $+u_{b} v_{b}\left(d_{b}-h_{b}\right)^{2}$ & $+2 u_{b} v_{b}\left(d_{b}+\left[u_{a}-v_{a}\right] m\right)\left(d_{b}-h_{b}\right)$ \\
4 & $u_{a} v_{a}\left(d_{a}+h_{a}+2\left[u_{b}-v_{b}\right] m\right)^{2}$ & $2 u_{a} v_{a}\left(d_{a}+\left[u_{b}-v_{b}\right] m\right)\left(d_{a}+h_{a}+2\left[u_{b}-v_{b}\right] m\right)$ \\
4 & $+u_{b} v_{b}\left(d_{b}+h_{b}+2\left[u_{a}-v_{a}\right] m\right)^{2}$ & $+2 u_{b} v_{b}\left(d_{b}+\left[u_{a}-v_{a}\right] m\right)\left(d_{b}+h_{b}+2\left[u_{a}-v_{a}\right] m\right)$ \\
& $+16 u_{a} u_{b} v_{a} v_{b} m^{2}$ & $+16 u_{a} u_{b} v_{a} v_{b} m^{2}$
\end{tabular}

Full complementary interaction $(d=h=m)$

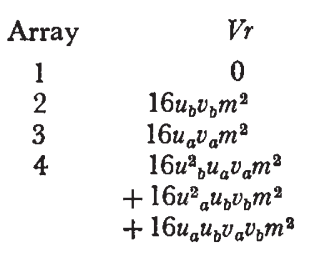

$$
\begin{gathered}
W r \\
0 \\
16 u_{a} u_{b} v_{b} m^{2} \\
16 u_{a} u_{b} v_{a} m^{2} \\
16 u^{2} u_{a} v_{a} m^{2} \\
+16 u^{2}{ }_{a} u_{b} v_{b} m^{2} \\
+16 u_{a} u_{b} v_{a} v_{b} m^{2}
\end{gathered}
$$

Full duplicate interaction $(d=h=-m)$

Thus the two types of interaction have characteristically different effects on the $W r / V r$ graph drawn from the results of analysis of a diallel cross. Complementary interaction of the type discussed gives rise to a curve which is concave upwards; duplicate interaction to a cluster of points at the origin (appearing to imply that the parents of these arrays are identical, though they may in fact have very different genotypes) while the point for the array 
of a parent carrying all the recessive alleles involved in this interaction, lies on the line of unit slope through the origin. The analogy between this latter situation, and the characteristic $\mathrm{F}_{2}$ ratios of $15: 1$ obtained in crosses involving two genes which show this relationship, is clear and interesting.

Various other, more complex, types of interaction have been examined for different gene frequencies. For less intense interaction of a complementary or duplicate nature than that discussed above, the curves described by Mather (1967) hold for equal gene frequencies. But for divergent

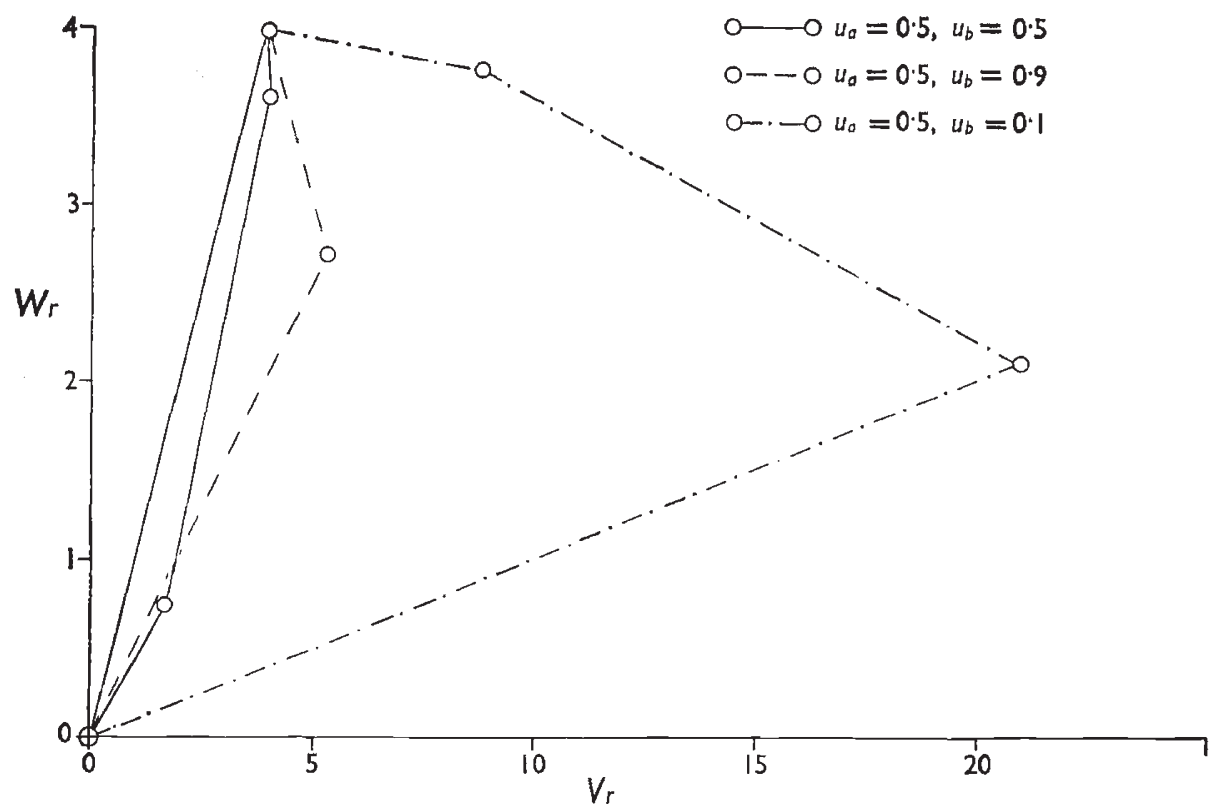

Fig. 1.-The $W r / V r$ graph for full complementary interaction (i.e. $d=h=m$ ) and varying gene frequencies. The distance between the points for arrays ( 1 ) and (4) has been standardised by varying the size of $m$. The line of unit slope is drawn for comparison (note the difference in scale of the $W r, V r$ axes). The points for arrays (1) and (4) lie on this line but those for arrays (2) and (3) lie below it and as $u_{a}, u_{b} \rightarrow 0$ the curvature becomes more pronounced.

frequencies, departures from these smooth curves are common, and the order of the points may be upset. For $i$ interaction alone, it is interesting to note that if $d=h=i$, a set of curves, concave upwards, is obtained as in the classical complementary situation involving all the interaction parameters. However the points for arrays (1) and (4) no longer lie on a line of unit slope. Thus, it is not possible to standardise the curves in the way used above. Curvature can nevertheless be shown to vary with gene frequency, and some of the curves obtained are shown in fig. 2. Fig. 3 shows the curves obtained when $d=h=-i$. Note that these are concave downwards as shown by Mather to be expected when gene frequencies are equal.

For $l$ interaction alone, it is interesting that whereas lines of unit slope are obtained where the gene frequencies are equal, this relationship does not hold for other gene frequencies: the situation is far more complex and confusing. The points do not necessarily lie either on a smooth curve, or indeed 
even lie in order relative to the $W r$ axis as in the preceding cases. The situation is equally confusing for $j$ interaction alone, even when gene frequencies are equal.

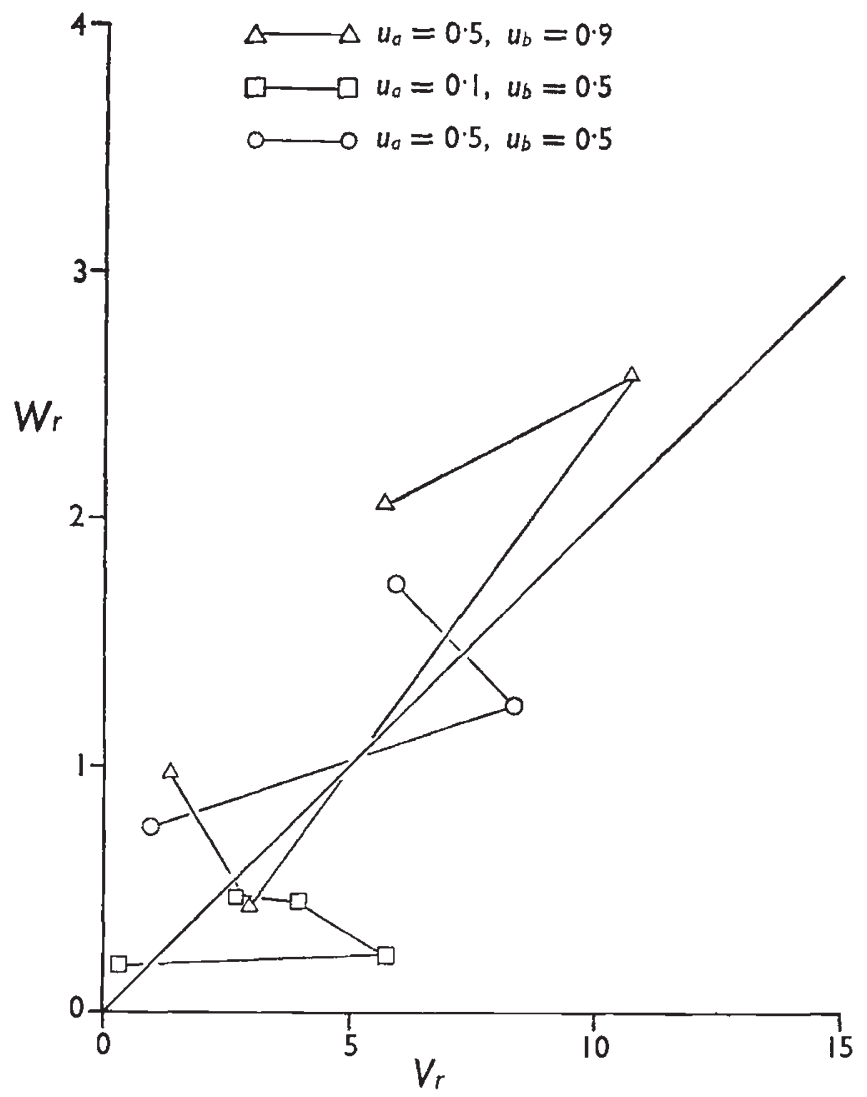

Fig. 2.-The $W r / V r$ graph for $d=h=i$ (other types of interaction absent) and varying gene frequencies. Curves which are concave upwards, as in the classical complementary situation (see fig. 1) are obtained. Again the degree of curvature varies with gene frequency. The line of unit slope through the origin is drawn for comparison.

\section{Gene association and dispersion}

With independence of distribution of the genes among the parents of a diallel cross, the expected frequencies of the parents $A A B B, A A b b, a a B B$, $a a b b$ are $u_{a} u_{b}, u_{a} v_{b}, v_{a} u_{b}, v_{a} v_{b}$ respectively. Diallel analysis is affected by departures from these frequencies caused by non-random distribution of the genes, i.e. association or dispersion.

Gene association and dispersion in diallels can be accommodated by the introduction of a parameter, $c$, such that the parental types $A A B B, A A b b$, $a a B B, a a b b$ occur with frequencies $u_{a} u_{b}+c, u_{a} v_{b}-c, v_{a} u_{b}-c, v_{a} v_{b}+c$. When the parents with reinforcing combinations of genes are in excess, i.e. the genes are predominantly associated, $c$ is positive. When parents with balancing combinations are in excess, i.e. the genes are predominantly dispersed, $c$ is negative. 
In the absence of non-allelic interaction the variances and covariances of arrays are as shown in table 6. There is rectilinearity of the $W r / V r$ graph only when $c=0$ : when $c \neq 0$, this rectilinear relationship is lost.

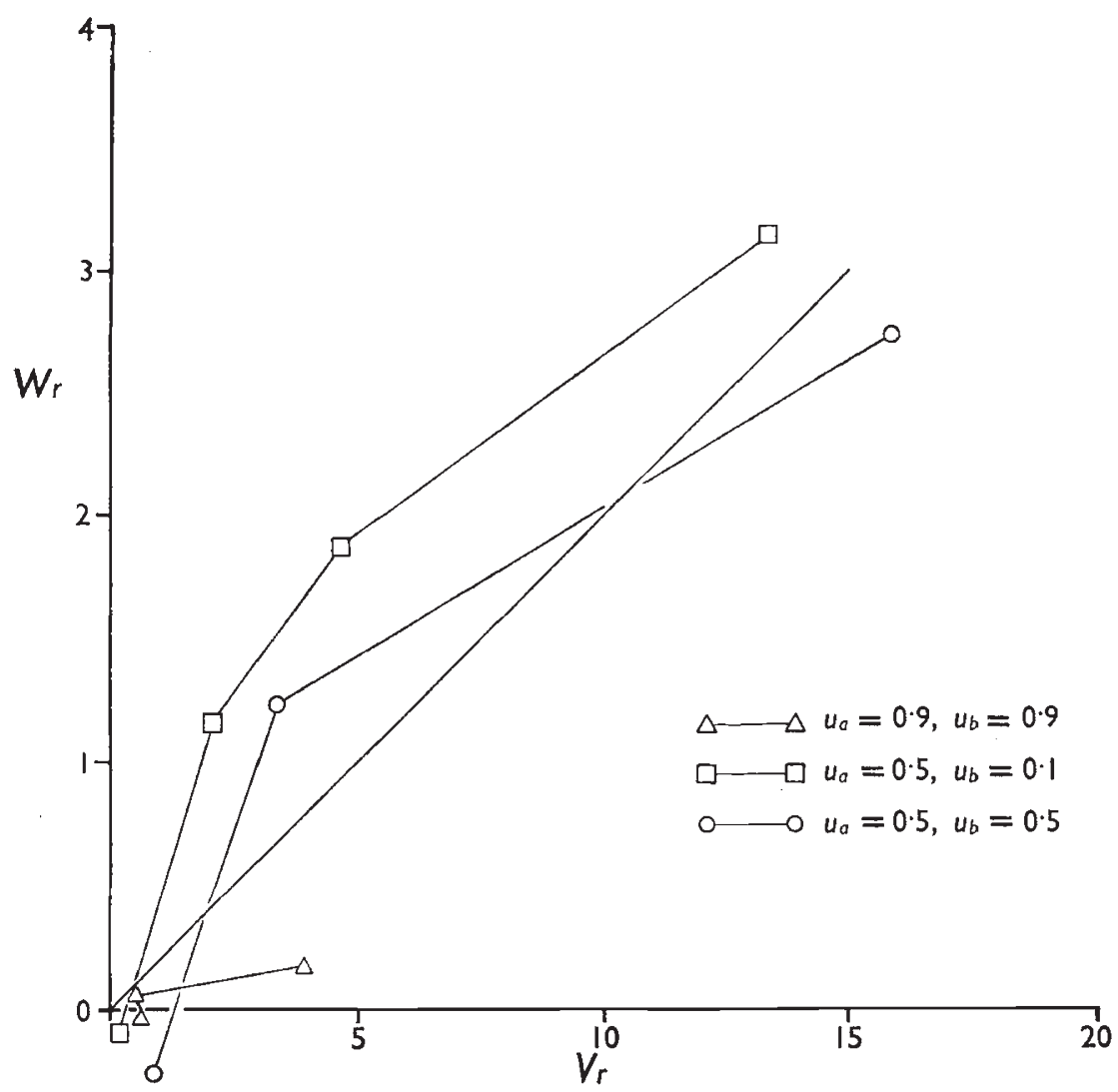

Fig. 3.-The $W r / V r$ graph for $d=h=-i$ (other types of interaction absent) and varying gene frequencies. The curves are convex upwards: again the line of unit slope through the origin is drawn for comparison.

Association and dispersion lead to displacement of the points for all the arrays from the line of unit slope obtained when these genes are independently distributed among the parents. However, for both arrays (1) and (4)

$$
W r-V r=u_{a} v_{a}\left(d_{a}^{2}-h_{a}^{2}\right)+u_{b} v_{b}\left(d_{b}^{2}-h_{b}^{2}\right)+2 c\left(d_{a} d_{b}-h_{a} h_{b}\right)
$$

And for both arrays (2) and (3)

$$
W r-V r=u_{a} v_{a}\left(d_{a}^{2}-h_{a}^{2}\right)+u_{b} v_{b}\left(d_{b}^{2}-h_{b}^{2}\right)+2 c\left(d_{a} d_{b}+h_{a} h_{b}\right)
$$

Hence the points for arrays (1) and (4) lie on a line of unit slope which intercepts the $W r$ axis at a point dependent both on $c$ and on the relative sizes of $d$ and $h$. The same is true of the points for arrays (2) and (3). This situation paradoxically results in an overall appearance of curvature of the $W r / V r$ graph, the direction and the degree of curvature depending on the sign of $c$, and on the size of $h_{a} h_{b}$, respectively. If $c$ is positive, i.e. there is F 2 
association of dominant genes among the parental lines, the line is convex upwards since the points for arrays (2) and (3) will lie on a line of unit slope to the left of that joining arrays (1) and (4). For dispersion of genes among the parents, i.e. $c$ negative, the line joining points (2) and (3) lies to the right of that joining points (1) and (4) (for $u_{a}=u_{b}=\frac{1}{2}$, and $d_{a}=d_{b}, h_{a}=h_{b}$, the points for arrays (2) and (3) will be equivalent). Hence the $W r / V r$ line is curved and concave upwards when the dominant genes are predominantly dispersed in the parental lines.

\section{TABle 6}

Variances and covariances for arrays of a diallel cross of four inbred lines where the genes are non-randomly distribute. among the parents (no interaction present)

\begin{tabular}{|c|c|c|c|c|}
\hline Array & Parent & Frequency & $V r$ & $W r$ \\
\hline 1 & $A A B B$ & $u_{a} u_{b}+c$ & $\begin{aligned} & u_{a} v_{a}\left(d_{a}-h_{a}\right)^{2}+u_{b} v_{b}\left(d_{b}-h_{b}\right)^{2} \\
+ & 2 c\left(d_{a}-h_{a}\right)\left(d_{b}-h_{b}\right)\end{aligned}$ & $\begin{aligned} & 2 u_{a} v_{a} d_{a}\left(d_{a}-h_{a}\right)+2 u_{b} v_{b} d_{b}\left(d_{b}-h_{b}\right) \\
+ & 2 c\left[d_{a}\left(d_{b}-h_{b}\right)+d_{b}\left(d_{a}-h_{a}\right)\right]\end{aligned}$ \\
\hline 2 & $A A b b$ & $u_{a} v_{b}-c$ & $\begin{aligned} & u_{a} v_{a}\left(d_{a}-h_{a}\right)^{2}+u_{b} v_{b}\left(d_{b}+h_{b}\right)^{2} \\
+ & 2 c\left(d_{a}-h_{a}\right)\left(d_{b}+h_{b}\right)\end{aligned}$ & $\begin{aligned} & 2 u_{a} v_{a} d_{a}\left(d_{a}-h_{a}\right)+2 u_{b} v_{b} d_{b}\left(d_{b}+h_{b}\right) \\
+ & 2 c\left[d_{a}\left(d_{b}+h_{b}\right)+d_{b}\left(d_{a}-h_{a}\right)\right]\end{aligned}$ \\
\hline 3 & $a a B B$ & $v_{a} u_{b}-c$ & $\begin{aligned} & u_{a} v_{a}\left(d_{a}+h_{a}\right)^{2}+u_{b} v_{b}\left(d_{b}-h_{b}\right)^{2} \\
+ & 2 c\left(d_{a}+h_{a}\right)\left(d_{b}-h_{b}\right)\end{aligned}$ & $\begin{aligned} & 2 u_{a} v_{a} d_{a}\left(d_{a}+h_{a}\right)+2 u_{b} v_{b} d_{b}\left(d_{b}-h_{b}\right) \\
+ & 2 c\left[d_{a}\left(d_{b}-h_{b}\right)+d_{b}\left(d_{a}+h_{a}\right)\right]\end{aligned}$ \\
\hline 4 & $a a b b$ & $v_{a} v_{b}+c$ & $\begin{aligned} & u_{a} v_{a}\left(d_{a}+h_{a}\right)^{2}+u_{b} v_{b}\left(d_{b}+h_{b}\right)^{2} \\
+ & 2 c\left(d_{a}+h_{a}\right)\left(d_{b}+h_{b}\right)\end{aligned}$ & $\begin{aligned} & 2 u_{a} v_{a} d_{a}\left(d_{a}+h_{a}\right)+2 u_{b} v_{b} d_{b}\left(d_{b}+h_{b}\right) \\
+ & \left.2 c\left[d_{a}\left(d_{b}+h_{b}\right)\right]+d_{b}\left(d_{a}+h_{a}\right)\right]\end{aligned}$ \\
\hline \multicolumn{3}{|c|}{ Variance of array means, } & \multicolumn{2}{|c|}{$\begin{aligned} V_{r}= & u_{a} v_{a}\left[d_{a}-\left(u_{a}-v_{a}\right) h_{a}\right]^{2}+u_{b} v_{b}\left[d_{b}-\left(u_{b}-v_{b}\right) h_{b}\right]^{2} \\
& +2 c\left[d_{a}-\left(u_{a}-v_{a}\right) h_{a}\right]\left[d_{b}-u_{b}-\left(v_{b}\right) h_{b}\right]\end{aligned}$} \\
\hline \multicolumn{3}{|c|}{ Covariance of array means, } & \multicolumn{2}{|c|}{$\begin{aligned} W_{r}^{-}= & 2 u_{a} v_{a} d_{a}\left[d_{a}-\left(u_{a}-v_{a}\right) h_{a}\right]+2 u_{b} v_{b} d_{b}\left[d_{b}-\left(u_{b}-v_{b}\right) h_{b}\right] \\
& +2 c d_{a}\left[d_{b}-\left(u_{b}-v_{b}\right) h_{b}\right]+2 c d_{b}\left[d_{a}-\left(u_{a}-v_{a}\right) h_{a}\right]\end{aligned}$} \\
\hline \multicolumn{3}{|c|}{ Mean variance of arrays, } & \multicolumn{2}{|c|}{$\nabla r=V_{r}^{-}$as above $+4 u_{a}^{2} v_{a}^{2} h_{a}^{2}+4 u_{b}^{2} v_{b}^{2} h_{b}^{2}+8 c^{2} h_{a} h_{b}$} \\
\hline \multicolumn{3}{|c|}{ Mean covariance of arrays, } & \multicolumn{2}{|c|}{$\overline{W r}=W_{r}$ as above. } \\
\hline
\end{tabular}

It should be noted that should $h_{a} h_{b}$ be negative the effect is to remove the points deriving from arrays (1) and (4) from their extreme positions in the $W r / V r$ graph and put them into the intermediate positions occupied by the points from arrays (2) and (3), the latter points in their turn taking up the extreme positions. Thus change of sign of $h_{a} h_{b}$ alters the relation of the arrays to the points on the $W r / V r$ diagram but it does not alter the overall pattern of the points and so has no effect on the relationship between the sign of $c$ and the type of curvature.

From table 6 it can also be seen that the difference in both $\mathrm{Vr}$ and $\mathrm{Wr}$ between the extreme arrays (4) and (1) is

$$
4 u_{a} v_{a} d_{a} h_{a}+4 u_{b} v_{b} d_{b} h_{b}+4 c\left(d_{a} h_{b}+d_{b} h_{a}\right)
$$

Thus the $W r / V r$ line is lengthened by predominant association ( $c$ positive) and shortened by predominant dispersion ( $c$ negative).

Fig. 4 illustrates the effects of association and dispersion of genes among the parents on the $W r / V r$ graph for the cases where $d=h$ (complete dominance) and where $d=2 h$ (incomplete dominance).

In the two-locus case, the effects of association and dispersion are equally pronounced, but for increasing numbers of genes, dispersion becomes of 
decreasing importance. Even if the genes are in a state of maximum dispersion, i.e. the parents most commonly used in the cross are of genotype $A b C d E$. . . etc. or $a B c D e$... etc., the effects of positive correlation between genes $A, C, E$ etc., and of negative correlation between genes $A$ and $B, C$ and $D$, etc. will tend to balance out. Complete dispersion is in fact impossible to achieve with more than two genes, just as is complete repulsion (see Mather,

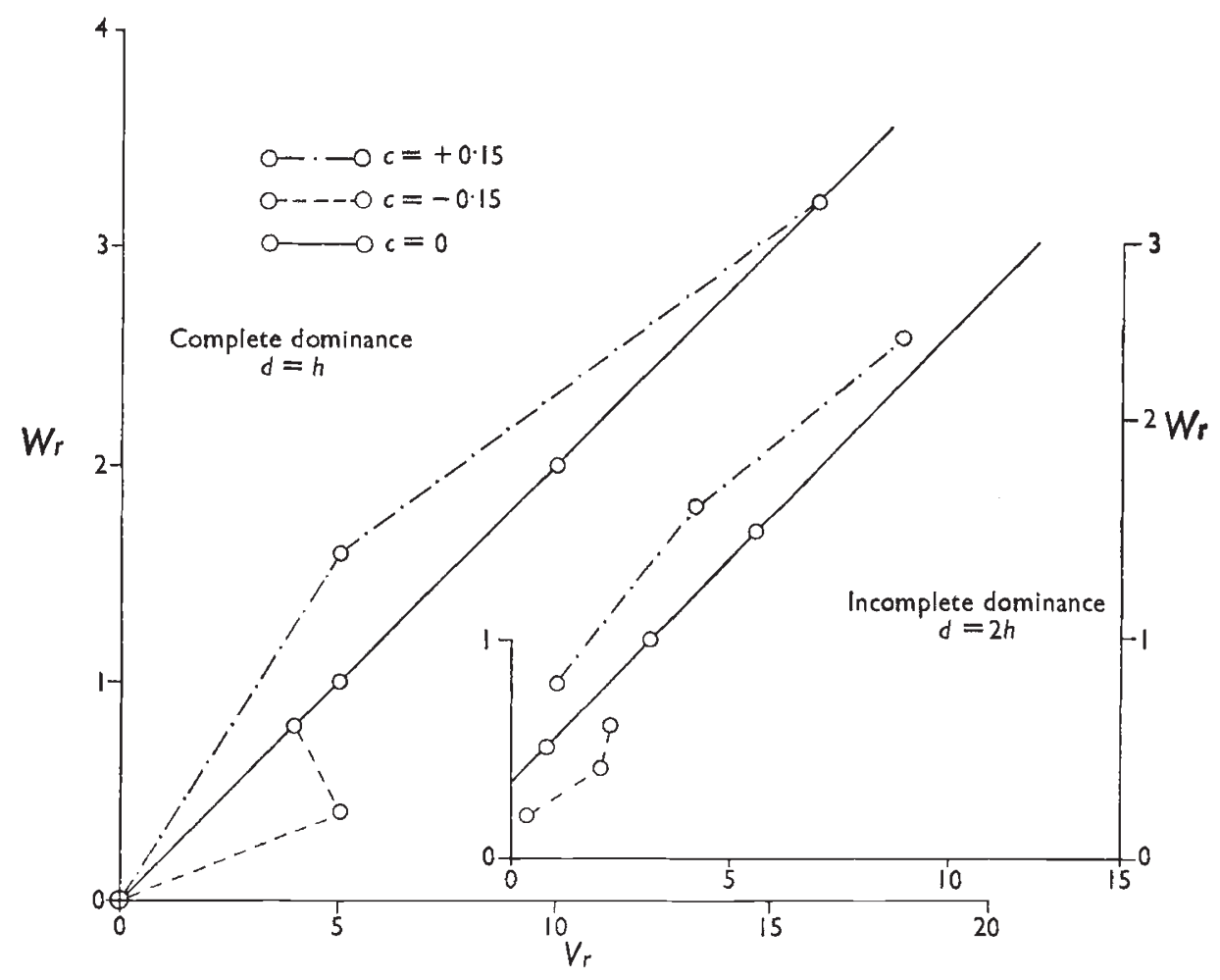

Fig. 4.-The effects on the $W r / V r$ graph of gene association and dispersion among the parental lines. The graphs are drawn for two levels of dominance, and three values of $c$. For complete dominance $(d=h)$ the points for arrays (1) and (4) lie on the line of unit slope through the origin for all values of $c$. For incomplete dominance (e.g. $d=2 h$, see graphs inset) and $c \neq 0$ these points lie off the line of unit slope drawn for $c=0$. Association of genes among the parents $(c>0)$ lengthens the line and gives a curve which is convex upwards. Dispersion gives a shortened line which is concave upwards.

1949), for the biometrical consequences of linkage). Association, on the other hand, where the parents most frequently used are of genotypes $A B C D E$. . . etc. and $a b c d e$... etc., can be complete with all the correlations between genes positive. With increasing numbers of loci, therefore, the effects of association become increasingly more noticeable. That dispersion in a system comprising a large number of genes, such as those controlling the numbers of sternopleural chaetæ in Drosophila melanogaster, is less noticeable in its effects on the $W r / V r$ graph than is association of such genes, has been shown experimentally to be the case by Hill (1964).

For all the expressions shown in table 6, the terms involving $c$ are independent of gene frequency and of simple form. This is the strength of using 
a parameter, such as $c$, to represent the situation where genotypes, but not individual genes, are non-randomly distributed among a population. The same situation could of course be expressed in various different ways, as noted by Hill (1964). The use of $c$, however, gives expressions for array covariances and variances which can be readily interpreted in terms of the effects of association and dispersion on the linearity of the $\mathrm{Wr} / \mathrm{Vr}$ graph.

Although the terms for array variances and covariances which involve $c$ are independent of individual gene frequencies, the possible range of $c$ is not: indeed the limits for $c$ are set by the contribution of individual gene frequences to the overall genotypic frequencies. For equal gene frequencies, i.e. $u_{a}=u_{b}=0.5, c$ must lie between -0.25 and +0.25 . In this situation if $c=+0 \cdot 25$, there is complete association of genes and only the extreme genotypes are present in the population. Equally, if $c=-0.25$, then the intermediate genotypes, only, are present.

For unequal gene frequencies, the possible range of $c$ is more limited. If positive, $c$ cannot exceed $u_{a} v_{b}$ or $v_{a} u_{b}$ whichever is the less; and, if negative, it cannot exceed $u_{a} u_{b}$ or $v_{a} v_{b}$, whichever is the less. Thus where gene frequencies are unequal, the effects of association and dispersion on the linearity of the $W r / V r$ graph are of less importance, though of course, association may still have important effects if a large number of genes are involved.

\section{Summary}

1. The effects of interaction, and of gene association and dispersion, on the $W r / V r$ graph of diallel analysis, are discussed for all gene frequencies.

2. Complementary interaction results in a $W r / V r$ graph which is curved concave upwards, the degree of curvature depending on the relative frequencies of dominant and recessive genes, and the intensity of the interaction. Full duplicate interaction results in grouping of points representing the arrays from parents bearing dominant genes at the origin irrespective of the gene frequencies; the points for arrays from fully recessive parents lie on a line of unit slope through the origin. The effects of other types of interaction are considered.

3. The effects of gene association and dispersion are considered in terms of a new parameter $c$. Association of similar genes among the parents results in a $W r / V r$ curve which is concave upwards, and dispersion a curve which is concave downwards. With more than two genes dispersion cannot be complete and its effects on the $W r / V r$ relations are thus weakened. Other effects of association and dispersion on the line are noted, and the use of $c$ as a parameter is discussed.

\section{References}

HAYMAN, B. I. 1954. The theory and analysis of diallel crosses. Genetics, 39, 789-809.

HAYMAN, B. I., AND MATHER, $\mathrm{K}$. 1955. The description of genic interaction in continuous variation. Biometrics, 11, 69-82.

HILL, J. 1964. The effects of correlated gene distributions in the analysis of diallel crosses. Heredity, 19, 27-46.

JINks, J. L. 1954. The analysis of continuous variation in a diallel cross of $\mathcal{N i c o t i a n a}$ rustica. Genetics, 39, 767-788.

MATHER, K. 1949. Biometrical Genetics. Methuen, London.

MATHER, K. 1967. Complementary and duplicate gene interactions in biometrical genetics. Heredity, 22, 97-103.

VAN DER VEEN, J. H. 1959. Test of non-allelic interaction and linkage for quantitative characters in generations derived from two diploid pure lines. Genetica, 30, 201-232. 